\title{
Reconstrucción de la justicia contractual desde la justicia relacional ${ }^{1}$
}

\author{
Reconstruction of Contractual Justice from Relational Justice
}

ROCÍO CARO GÁNDARA

UNIVERSIDAD de Málaga

Artículo recibido: 01 julio 2013

Solicitud de revisión: 08 octubre 2013

Artículo aceptado: 25 octubre 2013

\begin{abstract}
The reductionist view of contractual phenomenon, typical of civil codes and classical contract law, is a result of a blur that, overestimating the voluntary agreement, undervalues the exchange. Trying to show the partial and inadequate character of that vision of contract law, contextualists or relationists proposals have emerged, focusing on the defense of the relational contract model.These theories suggest relational justice as a new paradigm. It transfers the relational approach of sociology to law, and incorporates the analysis of plural economic reciprocity. Relational justice is formulated as a theory of justice (in the relationship), whose three elements (reciprocity, relatedness and sociality) offer a new theoretical framework where balances and imbalances that have been showing the legal contractual reality throughout history can be contextualized.
\end{abstract}

Keywords: contractual justice, relational justice, contract law, relational contract, essential contract theory.

Resumen

La visión reduccionista del fenómeno contractual, propia de los códigos civiles y del classical contract law, se produce como consecuencia de un desenfoque que, sobrevalorando el acuerdo de voluntades, minusvalora el intercambio.Tratando de evidenciar el carácter parcial e inadecuado de esa visión del Derecho contractual han surgido las propuestas contextualistas o relacionistas, orientadas a la defensa del modelo relacional del contrato. Estas teorías sugieren como nuevo paradigma la justicia relacional. La misma traslada al Derecho el enfoque relacional de la sociología e incorpora el análisis de la reciprocidad plural económica. La justicia relacional se formula así como una teoría de la justicia (en la relación), cuyos tres elementos (reciprocidad, institucionalidad y socialidad) ofrecen un nuevo marco teórico donde pueden ser contextualizados los equilibrios y desequilibrios que ha venido presentando la realidad jurídica contractual a lo largo de la historia.

1 Los argumentos esgrimidos en este estudio son desarrollados por la autora de forma bastante más extensa en Caro Gándara (2013: 53-115). 
Palabras clave: justicia contractual, justicia relacional, Derecho contractual, contrato relacional, teoría contractual esencial

\section{CRISIS DE LA JUSTICIA CONTRACTUAL: TOMA DE CONCIENCIA DOCTRINAL}

Las profundas transformaciones a las que se han visto sometidas las relaciones contractuales a lo largo del siglo $\mathrm{xx}$, caracterizadas por una fuerte intervención pública, impiden seguir considerando a la autonomía de la voluntad de las partes como fundamento de la fuerza obligatoria de los contratos, como había cristalizado en los códigos civiles decimonónicos, la mayoría de los cuales continúan vigentes en la actualidad. Dicha constatación llevó a algunos juristas a hablar de la muerte del contrato (Gilmore, 1974), o, al menos, de la crisis del mismo (Batiffol, 1968), que debemos entender como agotamiento del paradigma de la autonomía de la voluntad. ¿Se trata realmente de una muerte o, al menos, una crisis del contrato, o estamos ante una crisis más profunda, no del contrato, sino de la justicia (del contrato)? ${ }^{2}$ Pues debe tenerse en cuenta que las normas que regulan las relaciones contractuales no son neutras, sino que expresan los fines últimos del Derecho para cada legislador y en cada época (Savaux, 1997: 70 y s.; Adams y Brownsword, 1987: 205 y ss.; 2007).

Así, para las concepciones voluntaristas exacerbadas del contrato -que se fundamentan en las corrientes filosóficas basadas en la libertad y la racionalidad y en las teorías económicas clásicas y el utilitarismo-, los ajustes «espontáneos» del mercado, a través del juego de la oferta y la demanda, y la repartición de la riqueza que de ellos deriva, contienen en sí un principio de justicia ${ }^{3}$ que se concreta en la frase de Alfred Fouillée, filósofo francés (18381912): "qui dit contractuel, dit juste», y uno de cuyos máximos exponentes es el filósofo, jurista y economista de la escuela austríaca, F. A. Hayek. En ellas se equipara el interés general con la suma de los intereses particulares y se propugna que el control judicial debe quedar limitado a los consentimientos

2 El debate acerca de la justicia del contrato, que en este trabajo se aborda, queda muy lejos del debate filosófico-jurídico planteado acerca del desfondamiento de la justicia en general tras la crisis de la modernidad (Habermas, 1998a: 149 y ss.; 1998b: 571 y ss.; Habermas y Rawls, 1998). En el caso de la justicia contractual, como se sigue poniendo de manifiesto, son razones de índole práctica las que han mantenido este debate en términos reconducibles a la finalidad jurídico-económica que el contrato sigue llamado a cumplir en la vida social (Grossi, 2008: 138 y ss.).

3 Esa afirmación es completamente falsa y ofende la dignidad humana.Téngase en cuenta que el precio del grano de los países productores africanos se fija con años de antelación en la Bolsa de Chicago, antes de conocer el resultado de las cosechas, lo que en ocasiones provoca grandes hambrunas. 
de las partes. Estas concepciones presentan una versión «moderada», denominada «voluntarismo social», en virtud de la cual la fuerza obligatoria de los contratos deriva de la voluntad de las partes y de una norma jurídica superior a la que aquélla debe conformarse, pudiendo quedar limitada por el legislador, por referencia al orden público y a las buenas costumbres (Flour y otros, 2012).

Exacerbado o moderado, el voluntarismo, se caracteriza por el sacrificio de la justicia contractual en beneficio de la seguridad jurídica y la previsibilidad, que se concreta en el principio de intangibilidad de los acuerdos (sanctity of contract) y en el rechazo de la lesión como causa general de rescisión del contrato, de su revisión por concurrencia de causas imprevistas y de abuso de posición dominante y, en general, de la intervención judicial más allá del control de los consentimientos (Géraud-Llorca, 1994: 69 y ss.).

Frente a una lógica liberal, que afirma la autonomía de la voluntad y la primacía del mercado, se inscriben las doctrinas más sensibles a una necesaria conciliación entre autonomía privada e intervención pública, ante la constatación de la imposible realización de una auténtica libertad contractual, debido a la inexistencia de igualdad en las posiciones de las partes del contrato. En ellas se busca no la realización de la libertad, sino de la justicia, lo que exige una intervención judicial, no sólo para preservar el libre consentimiento de las partes, sino también la equidad de las estipulaciones y la equivalencia de las prestaciones. Ello supone un cambio en la consideración del contrato, dejando de entenderse como mero acuerdo de voluntades, para calificarse como instrumento de intercambio económico, lo que supone un nuevo enfoque, que pasa del acuerdo a la relación, adquiriendo así relevancia no sólo la fase de formación, sino también la de ejecución y la interpretación del mismo (Carbonnier, 2001: 288 y ss.; De Cossio, 1994: 33; Gete-Alonso, 2008: 129; Doral-García, 2011: 112-129).

En este contexto, se propugna que la intervención judicial se dirija principalmente al cumplimiento de nuevos deberes contractuales, como los deberes de información, transparencia, seguridad, lealtad, cooperación, renegociación, que están «enriqueciendo» el contenido obligatorio del contrato (Josserand, 1934: 340) y tienden a preservar el equilibrio contractual. En realidad tales deberes no son nuevos, pues se reconducen a la equidad y a la buena fe (Brownsword, 2006: 130 y ss.; Quiñones-Escámez, 2009:341 y ss.; Esteban de la Rosa, 2009: 405 y ss.), conceptos que habían sido elaborados por los jurisconsultos romanos, llegando hasta los códigos civiles decimonónicos (art. 1135 y 1134.3 Code civil, respectivamente, arts. 1255 y 1258 Código civil, 1374 Codice civile, $\$ 242 B G B$ ), pero una vez allí, corrieron distinta suerte. Desde el enorme desarrollo que han conseguido en la doctrina y 
la jurisprudencia alemanas, forzado por las crisis económicas derivadas de las dos guerras mundiales, hasta su olvido por la jurisprudencia francesa durante gran parte del siglo xx (Boudot, 2008: 646; Picod, 1993: 14 y ss;. 1989: 83 y ss.; 1988: 3318 y ss.; Mazeaud, 1999: 603 y ss.; 1997: 5 y ss.; Fabre-Magnan, 2001: 301 y ss; Chazal, 2003: 99 y ss.).

El debate que está teniendo lugar en el seno de la doctrina francesa desde hace más de un siglo (Savaux, 1997: 203; Rémy, 1987: 279) -más allá del movimiento solidarista (Grynbaum y Nicod, 2004) $-{ }^{4}$ y que se extiende a otros países (Ghestin, 2000: 90 y ss.), especialmente del ámbito anglosajón, ${ }^{5}$ nos lleva a reflexionar sobre los fines del Derecho y, más concretamente, del Derecho contractual. Según se le atribuya la función de preservar la seguridad jurídica o la justicia - conmutativa y equitativa-, se absolutiza o se relativiza la voluntad de las partes y, correlativamente, se limita o se amplía el juego de la buena fe (Cadiet, 2011: 177 y ss.). En efecto, ésta puede ceñirse a cumplir una función interpretativa de la voluntad expresa o implícita de las partes (Flour y otros, 2012) - sin que el juez pueda inmiscuirse más allá de la ley dada por ellas- o, por el contrario, intervenir para evitar el cumplimiento de un contrato manifiestamente desequilibrado o la aplicación de una cláusula abusiva bajo el pretexto del dogma de la intangibilidad de los acuerdos (Gete-Alonso, 2008: 124 y ss.). Por ello, se ha sostenido que no existiría la pretendida tensión entre seguridad jurídica y justicia contractual, si la primera, en lugar de entenderse como intangibilidad de los acuerdos -que, como tal, resulta un criterio rígido y fuente de injusticia-, pudiera elaborarse de manera más flexible, mediante la toma en consideración de todos los intereses en presencia, lo que exigiría establecer una jerarquía de valores, pues la única seguridad jurídica admisible es la que se fundamenta en la justicia (Ripert, 1925: 9 y ss.). ${ }^{6}$

4 Desde las primeras décadas del siglo xx se puso de manifiesto la necesidad de fundamentar «el contrato en la justicia y no la justicia en el contrato", así lo hicieron civilistas clásicos como Ripert (1925: 126); también, aunque desde una postura más próxima al solidarismo, Gounot (1912: 450).

5 Vid. infra.

6 En este sentido, el jurista francés Jacques Ghestin ha elaborado una teoría general del contrato que concilia interés particular e interés general, libertad, seguridad y justicia. Partiendo de la consideración del acuerdo de voluntades como elemento subjetivo esencial del contrato, concibe «lo útil»y «lo justo» como finalidades objetivas del mismo. Sustituye el valor de la voluntad de las partes por un valor intrínseco a la propia relación jurídico-negocial, que se corresponde con la función atribuida al Derecho de los contratos, de realización de la justicia y no sólo de la libertad. La utilidad y la justicia contractual constituyen para Ghestin los fundamentos últimos de la fuerza obligatoria de los contratos y de la teoría general de la que deriva todo su régimen jurídico. Igualmente constituyen los límites a la libertad individual de las partes, que no puede considerarse soberana, sino sometida a la ley, de la que ambos son auténticos principios inspiradores (Ghestin, 2000: 81; 1990: 147 y ss.; 1988 : 168 y ss.;1981: 35 y ss.). 


\section{JUSTICIA Y RELACIÓN CONTRACTUAL}

\subsection{Insuficiencia del Derecho contractual}

Una vez contextualizado el problema, llega el momento de abordar la cuestión principal de este estudio, que parte de la premisa de la insuficiencia del Derecho contractual, que supone asimismo una insuficiencia de la regulación jurídica del intercambio al que el contrato se refiere. Se trata de una insuficiencia de lo jurídico-normativo que, por afectar a la ordenación jurídica del contrato, hace resentirse también a éste. Pero esta insuficiencia no afecta necesariamente ni a la idea de justicia ni a la de relación "contractual», pues, en la medida en que corresponden a contextos más amplios, pueden incluso servir como referente para la superación de la aludida falta de capacidad normativa.

La insuficiencia del Derecho contractual se ha planteado especialmente en relación al llamado classical contract law. Ciertamente es una crítica en principio circunscrita al mundo jurídico anglosajón, pero, en cuanto lo que se rechaza es una visión excesivamente reducida del fenómeno contractual, se extiende a otras familias jurídicas, como acabamos de poner de manifiesto en el caso del Derecho francés (y adquiere todo su sentido en el intento de unificación europea de las distintas regulaciones nacionales del Derecho contractual). ${ }^{7}$ Más concretamente, la visión reducida o insuficiente del fenó-

7 Son tres las razones por las que dicha crítica debe entenderse referida a un ámbito más amplio, siendo la primera de ellas que el Derecho contractual clásico no constituye, como podría parecer, una realidad del pasado. Ciertamente el llamado classical contract law es un cuerpo normativo que se consolidó en la segunda mitad del siglo xix, como fruto de la labor de los tribunales de justicia (Stone, 2002: 1-2; ChenWishart, 2010: 11), pero sigue teniendo efecto en el Derecho inglés actual, en la medida en que las críticas doctrinales recibidas desde mediados del siglo $\mathrm{xx}-\mathrm{y}$ que han llevado en parte a que se hable del «moderno» o «neoclásico» Derecho contractual-, han producido cambios ciertamente, aunque, en general, no consolidados. Chen-Wishart (2010: 10 y ss.), habla en este sentido de valores en competencia, correspondientes al «clásico» y al «moderno» Derecho contractual, que siguen produciendo en éste una continua tensión. Además, en todo caso, los tribunales siguen aplicando el mismo lenguaje, las mismas categorías y estructuras del classical contract law (Stone, 2002: 2-3). A esto ha contribuido mucho el hecho de que, pese a algún intento serio de codificación (Chen-Wishart, 2010: 6), señala que en 1965, la Law Comission de Inglaterra, Gales y Escocia anunció un ambicioso plan para codificar el contract $l a w$, sin embargo ocho años después fue abandonado) el Derecho contractual sigue perteneciendo muy mayoritariamente al ámbito del common law-entendido en sentido estricto-, aun con reducidísimos ejemplos de legislación - p.ej., Misrepresentation Act 1967, Unfair Contract Terms Act 1977, Sales of Goods Act 1979, Contract (Rights of ThirdParties) Act 1999- (Beale y otros, 2010: 83). La segunda de las razones es que no sólo hablamos del Derecho contractual del Reino Unido, sino también de Estados Unidos y de otros países del ámbito anglosajón (Gilmore, 1995; Atiyah, 1990; Macaulay, 2003: 51-102; Macneil, 2003: 207-217). La tercera razón es que la insuficiencia del Derecho contractual objeto de crítica consiste en una visión del contrato reducida a los términos explícitamente pactados por las partes, como consecuencia de la importancia dada a la teoría de la autonomía de la voluntad - aunque se haya matizado que esta teoría sólo indirectamente penetró en el classical contract law (Beale y otros, 2010: 84) - y a la teoría económica de la rational choice (Campbell, 2011:9-15). 
meno contractual se produce como consecuencia de un desenfoque o de un enfoque desequilibrado que, sobrevalorando el acuerdo de voluntades, minusvalora el intercambio al que el contrato se refiere. En efecto, en relación a este segundo elemento, se ha querido recordar que el paradigma al que obedece el classical contract law también considera que el contrato «representa un "intercambio", por el que cada parte da algo en respuesta a la promesa del otro. Es la existencia de esta mutualidad (el efecto atribuido a través de la teoría de la consideration) lo que generalmente da lugar a la ejecutabilidad» (Stone 2002: 2). El intercambio representa así una oportunidad inexplorada de reforzar la justicia contractual. ${ }^{8}$ Por el contrario, se incide en el primer elemento (acuerdo de voluntades) y, en base al principio de libertad contractual, se considera a las partes soberanas para decidir los términos del contrato, siendo el principal papel del Derecho otorgar efecto jurídico a lo decidido por ellas. Debiendo ser interpretado de forma restringida dentro de las «cuatro esquinas del contrato»-según formulación que se ha convertido igualmente en clásica-, este enfoque reductivo se ve a su vez distorsionado por cuatro presunciones: $1^{a}$ ) que existe igualdad entre las partes, $2 .^{a}$ ) que la justicia del contrato existe en la medida en que ha sido negociado, 3 . $^{\text {a }}$ ) que los contratos son discretos (en el sentido de instantáneos, existiendo una posición de separación entre las partes y sin que tras el acuerdo exista -o sea relevante - un desarrollo posterior del contrato) y $4 .^{a}$ ) que los contratantes sólo actúan movidos por el propio interés (Chen-Wishart, 2010: 11; Stone, 2002: 2-3).

Tratando de evidenciar el carácter parcial e inadecuado de esta visión del Derecho contractual han surgido las propuestas contextualistas, o relacionistas, denominadas así por propugnar una ampliación de la visión del contrato, abarcando la relación contractual que constituye su contexto. En este sentido se enmarca la distinción propuesta por Macaulay entre real deal (verdadero contrato o relación contractual) y paper deal (documento escrito del contrato), que no refleja del todo las razonables expectativas de las partes, en la medida en que existe un «texto entre líneas», o unas «dimensiones implícitas», cuya toma en consideración se relaciona con una mayor justicia contractual, en la medida en que constituye un avance hacia la justicia am-

8 El intercambio es al mismo tiempo ocasión de equilibrio y justicia conmutativa y contexto de cooperación en clave de relación. Macneil, en este sentido, no niega la enorme contribución del classical contract law, sino que critica su compromiso tan parcial con la teoría de la voluntad, que excluye, como excepciones a la regla general, numerosos aspectos del fenómeno contractual: «si el papel del Derecho en la creación de contratos fuera presentado de forma más completa, esta distorsión no ocurriría, y estas materias serían vistas no como excepciones a la libertad de contratación sino como simple parte de la definición legal del contrato» (Macneil, 1960: 177). 
pliar los límites de la teoría de la rational choice (Macaulay, 2003:51 y ss.). También el jurista inglés Roger Brownsword ha avisado contra una consideración abstracta de la libertad contractual, no contextualizada en la verdadera relación y expectativas - razonables- de las partes, lo que puede llevar a sustentar una ética o ideología individualista, y no de cooperación (Brownsword, 2006: 28-30, 140-141). La relación contractual y la justicia vuelven a estar aquí, pues, del lado de lo que se pretende incorporar o recuperar (las dimensiones no explicitadas) para superar la insuficiencia del Derecho contractual. Nótese que se trata de una limitación del Derecho, no del contrato, al no reconocer aquél más que algunos pocos elementos del contrato.Y es que las dimensiones implícitas son directamente predicables del contrato, como pertenecientes a él. ${ }^{10}$ Por ello, la principal crítica que se hace al Derecho contractual clásico es no aceptar la existencia de un contenido duradero en los contratos, ni de una relación contractual, ni de su contexto social. ${ }^{11}$

9 El autor alude al hecho de que Shakespeare, en su obra El Mercader de Venecia, rehusó tener en cuenta las dimensiones implícitas del contrato, girando el argumento de la obra de forma principal -y asombrosa, por desencajada - en torno al derecho de Shylock, conforme al contrato, de extraer una libra de carne del cuerpo de Antonio, ante la imposibilidad por parte de éste de devolver el préstamo (Macaulay, 2003: 69-70). La visión de Macaulay es sin embargo muy diferente: considera extremadamente común que las relaciones comerciales sean de carácter continuado, y, además, entrelazadas entre sí y poseyendo sus propios sistemas normativos, por lo que el Derecho contractual debería incluir la dimensiones implícitas; en referencia a la mayor parte de los contratos, él los entiende como «acuerdos para cooperar» (Macaulay, 2003:80-81).Y, citando a Gordon (1985:565 y ss.) afirma que «las partes tratan sus contratos más como matrimonios que como estancias de una noche». Es importante añadir que, al igual que Macneil (vid. más adelante), Macaulay ha apoyado sus propuestas sobre la consideración relacional de los contratos en evidencias empíricas, consistentes en la contratación en base a la idea de cooperación y en el poco uso que las partes hacen de los remedios legales, tal como comenzó exponiendo en dos importantes publicaciones (Macaulay1963a:55 y ss.; 1963b: 13 y ss).

10 Se trata de dimensiones identificables de forma factible por parte de los tribunales. Así, por ejemplo, Campbell y Collins afirman que «los abogados aprecian que existe algo más para las relaciones contractuales que acuerdo y consideration» (Campbell y Collins, 2003: 25). John Wightman por su parte escribe que «son una parte vital de la maquinaria del Derecho contractual inglés existente» (Wightman, 2003: 145).Y menciona, concretamente: $1 .^{\circ}$ ) el lenguaje compartido y las instituciones sociales mínimas, $2 .^{\circ}$ ) el entendimiento entre las partes, que emerge con el tiempo, relativo al comportamiento mutuo, y $3 .^{\circ}$ ) la forma como se lleva a cabo la relación comercial en cada sector (Wightman, 2003: 147-148). Campbell y Collins mencionan como dimensiones implícitas las intervenciones equitativas del Derecho en referencia, en primer lugar, a la formación del contrato (relativas al control de la validez del consentimiento, a la producción o ausencia del mismo, a la consideration y al contexto del contrato); y, en segundo lugar, al contenido del contrato (términos implícitos por referencia a la costumbre y al uso, así como el conjunto de la matriz fáctica; igualmente aluden a principios como el trato justo, la buena fe o la cooperación (Campbell y Collins, 2003: 25-32).

11 I. R. Macneil defiende que el análisis de las transacciones debe siempre comenzar dentro de sus contextos y denuncia que el análisis del contract law es propenso a los prejuicios antisociales y, de hecho, esconde importantes aspectos como el poder y sus efectos (Macneil, 2003: 207-215). Para Collins, junto al contrato hay que tener en cuenta el contexto de la relación económica y de la relación de confianza, siendo dicho contexto el que puede explicar el poder discrecional presente en algunos contratos (Collins, 2003: 252). Günther Teubner incluso amplía las dimensiones implícitas 


\subsection{Contrato relacional y Teoría contractual esencial}

Podría presentarse a Ian Macneil como uno de los principales representantes - junto a Macaulay - del intento de evidenciar las dimensiones implícitas del contrato, propugnando la categoría del contrato relacional. Pero ello sería injusto: ${ }^{12}$ su gran aportación doctrinal es la construcción de una potente teoría, que él ha llamado essential contract theory, orientada a la defensa del modelo relacional del contrato, para conseguir así la superación de las estrechas miras del classical contract law. ${ }^{13}$

En efecto, Macneil empezó a medir el Derecho contractual con la realidad del intercambio, considerando que el primero no abarcaba el fenómeno que representa el segundo, caracterizado fundamentalmente por la cooperación entre las partes, conclusión a la que llegó a través de estudios empíricos (Macneil, 2003: 176 y ss.). ${ }^{14}$ Analizando el fenómeno de la cooperación, del

más allá de la intención de las partes y de su amplia relación, para abarcar las instituciones sociales en que sus contratos participan (Teubner, 2003: 362).

12 En efecto, no hace justicia a Macneil, quien lo considera entre los relacionistas o contextualistas en general. En la medida en que un grupo de autores ha defendido conjuntamente la valorización de las dimensiones implícitas del contrato, y ello haya provocado una consiguiente reacción doctrinal contraria, se ha podido profundizar indebidamente en la división en dos bloques supuestamente homogéneos. De dicho debate se hace eco, entre otros, Dori Kimel, quien alude al modelo relacional en tono crítico, considerándolo un único bloque identificado con la defensa de las dimensiones implícitas del contrato. Kimel plantea, de forma acertada, la necesidad de debatir en términos de razonabilidad, huyendo del extremismo, considerando obligado reconocer también algunas aportaciones aceptables de los relacionistas. Hay que decir por otro lado que Macneil (vid. infra) reconoce expresamente todo lo que de valioso ha aportado el classical contract law. Por ello debe entenderse menos justificado atribuir al mismo, en ese sentido, las críticas que en general puedan dirigirse a los defensores a ultranza de las dimensiones implícitas - como el propio Kimel hace-(Kimel, 2007: 233 y ss.). Macneil ofrece una teoría completa y compleja, basada en estudios empíricos - que responde también a una visión interdisciplinar sistemática- que le llevan a la identificación de sus famosas 10 categorías contractuales, las cuales pueden ser implícitas o explícitas, alejándose pues de las aludidas doctrinas relacionistas y, por tanto, queda lejos de merecer esas calificaciones. En el mencionado estudio Kimel argumenta, de forma principal, contra el que llama modelo contractual (en general), en base a la comparación con el régimen que habría que atribuir a la institución de la promesa, en una especie de reducción al absurdo, para el caso de que a la promesa se aplicasen los mismos postulados relacionales. Aparte de que la promesa puede no ser un buen término de comparación con el contrato, no es justo con Macneil lo que se expresa al final de dicho artículo, pues al tiempo que se le identifica con el modelo de defensa de las dimensiones implícitas, se renuncia expresamente a profundizar en sus propuestas concretas y se le atribuye la defensa de la supremacía del paradigma relacional de la teoría del contrato (Kimel, 2007: 255). En realidad, el paradigma que él propone es más amplio que el anteriormente utilizado por el classical contract law, al cual también incluye.

13 Aunque reconoce que su teoría es conocida ampliamente como teoría relacional del contrato, él la llama "teoría contractual esencial, una de las incontables teorías relacionales posibles del contrato" (Macneil, 2003:208). De ahí el título de este apartado, que alude tanto al «contrato relacional» como a su mencionada teoría.

14 En esta obra expresaba las cinco características principales del intercambio comercial, como resultado de su estudio empírico, siendo la primera de ellas la cooperación: $1 .^{a}$ ) cooperación, $2 .^{a}$ ) intercambio económico, $3 .^{a}$ ) plan para el futuro, $4 .^{a}$ ) potenciales sanciones externas y $5 .^{a}$ ) control y manipulación social. 
intercambio - más amplio que el del contrato-, como comportamiento social - en clave interdisciplinar-, indentificó diez categorías de cooperación ${ }^{15}$ que deben considerarse categorías del contrato - en la medida en que está imbuido en una relación (Macneil, 1983: 340-418). ${ }^{16}$

Tales categorías constituyen elementos o valores comunes del contrato, en base a los cuales Macneil explica el fenómeno de la contratación como intercambio, es decir, como contrato envuelto en una relación, ofreciendo un modelo contractual más amplio que el contemplado por el classical contract law. Su teoría requiere unas mínimas explicaciones complementarias: la primera, que atribuye mayor importancia a dos de dichas categorías en la dinámica contractual: la reciprocidad y la solidaridad (Macneil, 2011:311); la segunda, que continuamente se refiere a esas diez categorías como pertenecientes a dos dimensiones, es decir, son primero conducta - dimensión conductual- y después son norma, en la que se consolida dicha conducta - dimensión normativa- (Macneil, 2011:257); y la tercera aclaración consiste en que esas dos dimensiones, unidas, dan lugar a distintos tipos de contratos -según el grado de relacionalidad-, que pueden representarse en un spectrum de contratos en cuyos extremos existen dos polos opuestos: el contrato discreto y el contrato relacional; aunque en realidad Macneil afirma que el contrato discreto - es decir, en el que las partes permanecen totalmente separadas, realizando una transacción instantánea sin ninguna implicación

15 Así lo explica Macneil de forma retrospectiva (Macneil, 2011:366-367):

Empezando por el comportamiento animal y el del hombre primitivo percibí cuatro raíces primarias del contrato: (1) una matriz social, (2) especialización del trabajo e intercambio, (3) un sentido de elección y (4) consciencia del pasado, presente y futuro. Estas raíces por sí mismas, sin embargo, eran demasiado generales para resumir el contrato de una forma que pareciera útil. Por eso, intenté destilar lo que iba encontrando convirtiéndolo en un número manejable de categorías básicas de comportamiento a partir de dichas raíces. Como el comportamiento humano repetido invariablemente crea normas, estas categorías de comportamiento son también categorías normativas.

En realidad, es algo más amplia la forma como el autor explica estas diez categorías comunes del contrato, partiendo de la sociedad - que construye el «edificio del contrato», cuyo papel tiene lugar en tres niveles: el primer nivel es el de las relaciones sociales ontológicamente fundamentales en las que está inserto el ser humano, la estructura de los significados compartidos, el lenguaje, la normatividad, etc.; el segundo nivel, la política de fondo de la sociedad burguesa (denominando background social matrix a la suma de estos dos niveles); el tercer nivel se compone de normas externas (impuestas no sólo por el Derecho positivo, sino por los grupos sociales, las costumbres, usos, etc.) y las normas internas (íntimamente ligadas a las externas) y que se concretan en las diez categorías contractuales (Campbell, 2011: 12-15).

16 Las diez categorías son las siguientes: $1 .^{\circ}$ ) integridad de rol, $2 .^{\circ}$ ) reciprocidad, $3 .^{\circ}$ ) ejecución del plan, $\left.4 .^{\circ}\right)$ prestación del consentimiento, $5 .^{\circ}$ ) flexibilidad, $\left.6 .^{\circ}\right)$ solidaridad contractual, $7 .{ }^{\circ}$ ) restitución, confianza y protección de las expectativas (normas vinculantes), $8 .^{\circ}$ ) creación y restricción de poder, $9 .^{\circ}$ ) adecuación de medios y $10 .^{\circ}$ ) armonización con la matriz social (es decir, con la norma supracontractual). 
relacional-, sólo existe de forma ideal, por lo que él lo llama as-if-discrete (Campbell, 2011: 28).

En los últimos años Macneil insiste en la neutralidad de su teoría, que debe ser considerada tan sólo una herramienta de análisis, no necesariamente predispuesta - aunque tampoco cerrada - a la intervención del Estado en las relaciones contractuales, a la cooperación o a valores sociales, comunitarios o humanitarios (Macneil, 2003: 207); al tiempo que evidencia que son las concepciones «discretistas» - basadas en la visión clásica del contract law y rational choice - las que no pueden considerarse neutrales, por no tener en cuenta los diversos elementos de la relación contractual, sino una parte muy reducida de ellos. ${ }^{17}$ Hace más de una década, haciendo gala de su objetividad sobre el grado de impacto de su teoría, se mostraba satisfecho con la frase "iahora ya todos somos relacionistas!», a la vez que reconocía la ausencia de unanimidad a la hora de aplicar esa consideración relacional al Derecho contractual. ${ }^{18} \mathrm{Y}$, de hecho, las aportaciones de Macneil han tenido un significativo impacto -ciertamente no general- en la comprensión y en la docencia del Derecho contractual en Estados Unidos -y, en menor medida, en el Reino Unido-, (Feinman, 2011:59-66; Vincent-Jones, 2011: 67-85) a lo que ha contribuido la popularización de su «Casebook» (Macneil y Gudel: 2001).

Macneil no plantea directamente una teoría de la justicia, sino del contrato. No obstante, su enfoque relacional lleva a incluir en segundo plano implicaciones de justicia, vinculadas precisamente a la relación. Aparte de alguna semejanza con el enfoque de justicia relacional (ver apartado siguiente, en relación a las tres explicaciones complementarias anteriores), cabe destacar, entre los elementos o valores comunes del contrato, el último de ellos, que consiste en la armonización con la matriz social; o su idea de intercambio (unida al spectrum de contratos), que expresa una combinación de elementos discretos y relacionales, en coherencia con lo que él llama «irracional esquizofrenia en el centro de la naturaleza humana», según la cual el ser humano es, al mismo tiempo, totalmente individualista y totalmente comunitario (Macneil y Gudel, 2001: 51-52); por lo que propugna, para su propia supervivencia, como principios de comportamiento, las dos categorías

17 «La teoría contractual esencial, una de las incontables teorías relacionales del contrato, es una herramienta neutral de análisis, no orientada hacia visiones sociales particulares. El hecho de que a los discretistas les parezca que favorece las visiones humanitarias es justamente el producto de la no neutralidad de su propia herramienta de análisis: teoría de la rational choice y classical contract law» (Macneil, 2003: 217).

18 El 29 de enero de 1999 tuvo lugar en la Facultad de Derecho de la Northwestern University, donde Ian Macneil había sido docente desde 1980, un simposium en su honor. Cuando publicó las respuestas a las observaciones que se le habían hecho a su trabajo, añadió al final un apartado V, titulado «Symposium Postscript», que a su vez contenía dos apartados: el apartado A, titulado «We're all relationists now!»; y el apartado B, denominado «But not when it comes to law» (Macneil, 2011:383-84). 
contractuales principales: solidaridad y reciprocidad; la reciprocidad -obtener algo en respuesta a dar algo- reduce la tensión en una criatura que es a la vez individual y social, en tanto que la solidaridad permite la proyección de la reciprocidad en el tiempo (Macneil, 2011:312).

\subsection{El enfoque de justicia relacional}

La teoría contractual de Macneil nos conduce de lleno al enfoque de justicia relacional. Éste analiza la presencia o ausencia de justicia en la relación jurídica. Parte de la estructura de la relación social y sus componentes, integrando la realidad jurídica en el ámbito interdisciplinar de la socialidad. Para ello traslada al Derecho el enfoque relacional de la sociología (Donati, 2006) e incorpora el análisis de la reciprocidad plural económica (Bruni, 2011 y 2006). Puede sintetizarse en los siguientes puntos (Márquez-Prieto, 2012, 2010 y 2008):

1. ${ }^{\circ}$ La idea de justicia encuentra explicación desde la noción de relación, entendida como vínculo recíproco, es decir, vinculación y a la vez reciprocidad (sus dos ejes), siendo la justicia su efecto emergente. No es una idea ajena a las categorías concretas de justicia -distributiva o conmutativa-, a las cuales necesariamente ha de incluir. Pero, en la medida en que se actúe de buena fe -o equitativamente- con la otra parte, y en la medida en que el contrato -e incluso su contexto legal- exprese contenidos de justicia, ello producirá un efecto en la propia relación jurídica (justicia relacional). ${ }^{19}$

2. ${ }^{\circ}$ La morfogénesis de la justicia relacional tiene lugar a través de una dinámica bilateral, no sólo entre los sujetos, sino también en torno a los dos ejes o componentes de la relación: el eje normativo o de vinculación (la parte estructural, institucional, obligatoria, normativa), y el eje de reciprocidad (la parte axiológica-conductual; valores, pero en la medida en que se traducen en comportamiento u omisión; la

19 Se contempla la posibilidad, pero no seguridad, de que exista y se perciba justicia en la relación. Ello se corresponde, además, con la opción tradicional del Derecho, que parte de la necesidad y de la posibilidad de actuar de buena fe. Es decir, el Derecho no sólo se ha considerado tradicionalmente a sí mismo como un método de solucionar conflictos, sino también como un instrumento de regulación de la conducta y de construcción de expectativas (Teubner, 2005:133). La justicia conmutativa es un dar de forma equivalente; la justicia distributiva es dar a cada uno lo que es suyo. En ambos casos hay un sentido obligado. La justicia relacional exige cumplir con ambos tipos de justicia, pero, además, significa apuntar a la relación, mediante una actuación equitativa, y conforme a la buena fe, ya sea por obligación o por liberalidad. El efecto que esa forma de actuar produce en la relación es la justicia relacional. 
parte ágil, libre, viva). ${ }^{20}$ Ambos ejes pueden adaptarse entre sí en mayor o menor grado. Recuérdese que Macneil afirma que los valores o categorías contractuales son en primer lugar, comportamiento o conducta y, posteriormente, norma. Y que su spectrum de contratos va de menor a mayor grado de relacionalidad. De la misma forma, el enfoque de justicia relacional toma en consideración esas dos dimensiones, que Macneil llama conducta y norma (si bien llamándolas reciprocidad y vinculación), pero de forma separada (en dos ejes o ámbitos). En realidad no se presentan de forma separada, pero separarlos (artificialmente, teóricamente) permite comprender la dinámica relacional (que exige tomar una distancia). Lo normal es que la propia relación jurídica parezca invisible (es el tercer elemento; $v i d$. infra) precisamente porque se suele pensar lo jurídico sólo en clave normativa (elemento estructural) dejando fuera la reciprocidad (elemento dinámico). ${ }^{21}$ Conviene también aludir, por la semejanza con esta dinámica bilateral entre los dos aspectos de la relación, a la complementariedad que para Macneil existe entre los que denomina principales valores comunes del contrato (vid. supra): reciprocidad y solidaridad: la reciprocidad (dar un valor, a cambio o en espera de recibir otro, directa o indirectamente), promueve la solidaridad (la confianza, la unidad), lo que hace a la reciprocidad sostenible a largo plazo (Macneil, 2011: 298-299, 311).

3. ${ }^{\circ}$ Dentro de la propuesta de la justicia relacional debe insistirse en el hecho de que la relación (jurídica), en sí misma considerada, constituye el tercer elemento, denominado socialidad, porque alude no sólo a la relación (en la medida en que se va continuamente haciendo), sino a todas las relaciones interconectadas, y a la inercia, cultura, valores del grupo o del contexto social. Por eso la relación (o socialidad) no sólo es, de los tres elementos de cada escena, en el que resul-

20 El eje o ámbito de institucionalidad se denomina así porque (estructural, institucional, legal o formalmente) establece o sustenta la relación. El eje de reciprocidad es la dinámica de interacción libre, el primer paso o primera fase, que, en la medida en que se consolida, se convierte en institucionalidad; es el espíritu, sin el cual la institucionalidad es letra muerta. Cada uno de esos ejes es lo que «no es» el otro, al cual dan sentido.

21 «[... si la institucionalidad no está abierta a la reciprocidad, se queda en unilateralidad. En puridad, ello no significa que para el Derecho la relación no exista. Pero la considera unilateralmente, viendo en ella sólo su contenido, de manera unidireccional: el derecho de uno respecto del otro; el deber de uno para con el otro. No ve la relación interactiva. Para ello es preciso introducir verdaderamente el enfoque de reciprocidad: el juego continuo y dinámico entre los sujetos de la relación, es decir, la relación propiamente. Eso hace que también lo relacional y lo recíproco se autoadapten, puesto que la reciprocidad no es sólo un aspecto de la relación, sino el aspecto que hace que se viva la reciprocidad entre los dos aspectos. Y, de forma paralela, la institucionalidad hace que la reciprocidad no sea sólo motivación, sino también responsabilidad» (Márquez Prieto, 2012: 65). 
ta más humanamente perceptible la justicia (siendo en él donde la misma emerge o no), sino que al mismo tiempo se convierte en criterio crítico de la misma, pues la socialidad es el contexto propio de los valores de justicia. También en la teoría de Macneil está presente - con mayor o menor evidencia- este tercer elemento. En la medida en que identifica, como décimo valor contractual, la armonización del contrato con su matriz social, afirma que la relación es una «minisociedad» (Campbell, 2011:18); y ya desde los primeros pasos de su teoría, en 1960, afirmaba que «el contrato es un edificio parcialmente construido por las partes pero también parcialmente construido por la sociedad, por el derecho» (Macneil, 1960: 177). Frase feliz en la que, realmente, se mencionan - aun sin pretenderlo- los tres elementos del enfoque de justicia relacional: reciprocidad, socialidad e institucionalidad o vinculación, por este orden.

\subsection{Una ¿nueva? teoría general de la justicia contractual}

En este momento de la exposición podríamos unir a la búsqueda de un nuevo paradigma contractual la necesidad -también apuntada en el debate sobre un Derecho contractual europeo (Lurger, 2005: 442 y ss.; 2011: 335 y ss.)- de disponer de una teoría general del contrato. En tal sentido, y en atención a las propuestas doctrinales comentadas, que sugieren claramente como nuevo paradigma el enfoque relacional, surge la cuestión de si la teoría de la justicia relacional puede contribuir a iluminar y concretar esta opción. Siendo la respuesta concreta que este enfoque, que participa del paradigma relacional, manifiesta dos importantes características: la primera, que se formula ante todo como una teoría de la justicia (en la relación), lo que constituye la identificación de una referencia en torno a la cual pueden ser orientados los elementos esenciales del debate; y la segunda, que la definición de los tres mencionados ámbitos (reciprocidad, institucionalidad y socialidad) ofrece un marco teórico donde puedan ser contextualizadas (y reenfocadas) las argumentaciones jurídicas diversas al respecto, de forma suficientemente compatible. $^{22}$

22 No es una teoría del Derecho, ni una teoría del contrato. Ni siquiera es una teoría de la relación, aunque es la relación el paradigma en el cual se apoya, sino, ante todo, de la justicia, analizada en la relación. La justicia, pues, se convierte en referencia de los distintos elementos del contrato, los cuales, ensanchados al entorno de la relación contractual, se ordenan en función de los tres mencionados ámbitos. Al componer éstos un marco ancho, dejan espacio para las diversas argumentaciones jurídicas convencionales (de forma compatible, al no vibrar en la misma longitud de onda), cada una en su justo lugar, que quedan así convenientemente contextualizadas. 
Esto se dice desde el convencimiento de que una concepción de la justicia en la relación, en base a la distinción de los tres mencionados ámbitos - la propia relación y sus componentes - ha estado presente en la realidad jurídica del contrato desde sus inicios, habiendo presentado a lo largo de la historia equilibrios o desequilibrios, que pueden reinterpretarse ahora desde este nuevo enfoque, desde el que recomponer aquí la historia del Derecho de los contratos a grandes rasgos.

El primer escenario corresponde al período que podría ser denominado «construcción (del contrato y de la justicia del contrato)», en el cual, efectivamente, el Derecho romano hizo surgir el contrato como expresión negocial de justicia. Ya Mucio-Escévola entendía el contrato como combinación de dos partes o aspectos (es decir, no sólo un trato entre dos sujetos, sino una conexión entre dos elementos o categorías materiales: emptio-venditio, locatio-conductio) entre las cuales existía un nexo o elemento latente, una regla o principio normativo, que regulaba de modo unitario la conexión entre esas dos partes (siendo importante que la regla surgía de los intercambios comerciales). Y también esa estructura triangular se observaba en la alusión a los negocios ex bona fide, puesto que la determinación de la existencia o no de un verdadero acuerdo negocial (elemento de institucionalidad, diríamos), o la existencia de un error que lo viciase, habría de ser determinada por el pretor a través de la interpretación procesal de la conducta de las partes (es decir, examinando la reciprocidad), tomando como referencia la buena fe (ligada a las relaciones de intercambio, es decir, socialidad). Así, la fuerza obligatoria del contrato aparecía ligada, no al contrato mismo -institucionalidad-, sino a la buena fe, a la confianza propia de las relaciones sociales -socialidad-(Schiavone, 2005:155-197). Esto también significaba conectar la fuerza obligatoria con la justicia; de ahí el nombre dado al tratamiento de este período: construcción del contrato y de la justicia contractual.

En cuanto a la definición de los tres aspectos de la justicia relacional, se dio un paso importante con Labeón. En efecto, si pareció más evidente, desde un principio, que era la socialidad la que podía aportar el criterio, a la vez, de lo justo y lo obligatorio, Labeón ayudó a la mejor identificación de los dos componentes de la relación contractual: a la idea de reciprocidad, presente en el synallagma griego, añadió el vínculo romano de la ob-ligatio, denominando consecuentemente a la relación contractual «vínculo recíproco» (pudiéndose ya reconocer la identificación de la institucionalidad - o vinculación - y la reciprocidad) consagrado en su fórmula ultro citroque obligatio (Schiavone, 2005: 269-292). 
La insistencia de Ulpiano en el elemento causal del contrato afianza la idea de una reciprocidad orientada a la institucionalidad. El hecho de concretar la causa en la prestación efectiva, ya realizada, por una de las partes, de aquello a lo que ambas se han obligado, sugiere considerar la reciprocidad como primer paso efectivo -y causa- de la ejecución completa del contenido del contrato, conforme al acuerdo pactado (institucionalidad). Se advierte en la idea de causa una concepción de justicia (de hecho se la conecta con la protección mediante una actio), por la estrechísima conexión entre la manifestación de esa conducta de reciprocidad (prestación efectuada) y el acuerdo de voluntades (Schiavone, 2005:341-360). Pero para Labeón y para Ulpiano, como había comenzado siendo para Escévola, era necesario proteger la confianza - fides- de las operaciones (Labeón), la fiabilidad de las relaciones civiles (Ulpiano). Una buena fe en definitiva que, desde un principio, transmitía socialmente la persona del paterfamilias ${ }^{23}$ y que, en referencia a las relacionales sociales (socialidad), se fue materializando, ligada a la equidad, en la fórmula ex aequo et bono. La socialidad presente en la concepción jurídica romana -injustamente considerada individualista- era mucho más rica que los frutos que de la misma han trascendido (Schiavone, 2005:361-399), a pesar de que la buena fe y la equidad se siguieron utilizando como fuente de soluciones de justicia para adaptar el acuerdo contractual y su contenido a diversas vicisitudes y eventualidades, como fueron poco después importantes ejemplos la reductio ad aequitatem o la cláusula rebus sic stantibus, propugnadas por glosadores y comentaristas medievales (Chamie, 2012: 219 y ss.; Gordley, 2004: 513; De Cossio, 1994: 17 y ss.).

El segundo de los escenarios sería el de la fase de deconstrucción, que podría ser definido como el de la «exaltación y crisis de la libertad individual decimonónica» en la que cristalizan las influencias de los filósofos racionalistas, los economistas clásicos y del utilitarismo, de los siglos XVII y XVIII. Esa denominación pretende expresar el hecho de que la libertad individual no es, en realidad, el valor tan supuestamente respetado en esa fase, caracterizada por la codificación y por un desequilibrio a favor del ámbito de institucionalidad. Efectivamente, es el aspecto legal el que -con sometimiento de la jurisprudencia y de la costumbre-, al tiempo que define los elementos esenciales del contrato y predica del mismo su valor de ley, lo deja en realidad amordazado en su «intangibilidad»o «santidad», sin permitir en general adaptaciones posteriores sino por razones muy graves. ${ }^{24}$ Es decir que, aun

23 También en este sentido Alain Supiot reivindica - en el siglo xxI- el valor incalculable de la confianza en la palabra, es decir, en el trato humano, en la interacción personal y directa, que genera una dinámica (social) que no puede ser sustituida por el contrato ni por la ley (Supiot, 2005: 174-175).

24 El art. 1134 del Code civil francés de 1804, en cuyo párrafo tercero exige que todos los contratos 
partiendo de la idea de justicia, como superior a la libertad contractual, y declarando expresamente el necesario tributo - pero inoperante- a la equidad y a la buena fe, en realidad evidencia (de forma paradigmática en el caso francés) una libertad meramente formal, y por tanto, una injusticia relacional por desigualdad material. El enfoque de la justicia relacional ayuda a ver más claro que cuando el Derecho, como institucionalidad, debilita sus lazos con la reciprocidad (libertad plural, interacción viva) y la socialidad (justicia), es el propio Derecho el que se debilita. Sintiéndose suficientemente legitimado para atribuir por sí mismo fuerza de obligatoriedad, se convierte en realidad en vasallo del totalitarismo ideológico o económico.Y, en cuanto al contrato, este peso excesivo de la institucionalidad, de lo normativo, hace siempre sospechar si no está sirviendo a una sola de las partes: a aquélla a quien, por su posición socioeconómica, le basta la igualdad formal. Es, después de todo, la misma trampa, para la justicia en el contrato, que esconde la teoría de la rational choice, como han intentado señalar los relacionistas y contextualistas -al incorporar las dimensiones implícitas del contrato- y, singularmente, Ian Macneil-quien pone al descubierto el carácter parcial de dicha teoría, no sólo porque parte de una visión reducida del contrato (ignorando su aspecto relacional, su socialidad), sino porque da pie a una reciprocidad interesada. Precisamente, como han puesto de manifiesto, con base en la teoría de juegos, Brownsword (2006: 129) - entre los juristas - y Bruni (2012: 73) - entre los economistas- es otro tipo de reciprocidad, el de la cooperación, el que promueve mayor riqueza y justicia social.

El tercer y último escenario, relativo a la fase de «reconstrucción», representa los diversos intentos de superación del desequilibrio anterior, a los que se pretende aludir con la mención «de la justicia contractual a la justicia relacional del contrato». En esta fase también existe una cierta llamada a la modestia para el ámbito de institucionalidad, sugerida en el apartado sobre la «insuficiencia del Derecho contractual», donde dicho bloque normativo se enfrenta a sus limitaciones reguladoras, no sólo por no atender con éxito a la relación contractual en su totalidad, sino sobre todo por la necesidad de superar la diversidad legislativa que representan los distintos ordenamientos jurídicos. Ciertamente el proceso de unificación del Derecho contractual europeo constituye, por sí mismo, una oportunidad de continuar esta reconstrucción. Los mencionados intentos están suponiendo de una forma muy evidente la recuperación del ámbito de socialidad, en la medida en que las

hayan de celebrarse de buena fe, no ha sido aplicado por la Cour de Cassation hasta 1985. Ese retraso de casi dos siglos ha sido consecuencia de la interpretación liberal e individualista que del mismo ha imperado durante los siglos xix y gran parte del xx (Géraud-Llorca, 1994: 69 y ss.; Chazal, 2003: 99 y ss.). 
cláusulas generales - primero en Alemania, ${ }^{25}$ pero también en otros países, no sólo de Derecho continental-, conectan con la buena fe, las buenas costumbres, la equidad o los usos sociales y comerciales (Jauffret-Spinosi, 2006: 23 y ss.; Bigwood, 2003; Kartwright, 1991; Capper, 1998: 479 y ss.; Posner, 1995:283; Volpe, 2004: 35 y ss.). Incluso estas cláusulas generales están dando lugar - principalmente en los proyectos europeos de Derecho uniforme - a nuevos deberes de cooperación, transparencia o lealtad ${ }^{26}$ (Schulze, 2008: 177 y ss.; Gómez-Pomar y Gil-Saldaña, 2012; Hartkamp, 2011: 239 y ss.; Twigg-Flessner, 2008: 97 y ss.; Basozábal-Arrue, 2012: 181 y ss.).Y no sólo en los aludidos textos europeos, igualmente en un contexto más amplio como el de Naciones Unidas (Principios UNIDROIT) ${ }^{27}$ y en las propuestas de reforma de los Derechos nacionales - principalmente en Francia y España (Picod, 2009; Doral, 2011: 146 y ss.; Bosch-Capdevila, 2012: 365 y ss.; Salvador-Coderch, 2009: 17 y ss.)-, se advierte de forma muy pujante la necesidad de preservar la justicia como equilibrio, no sólo en su momento inicial, sino durante el resto de la vida funcional del contrato.

\section{CONCLUSIONES}

Estamos, pues, ante una recuperación muy prometedora de la socialidad, que abre una gran oportunidad para la justicia relacional. Incluso sería deseable un mayor desarrollo del ámbito de reciprocidad, puesto que la promoción adecuada de la socialidad habría de suponer también un incentivo de este otro aspecto, componente de la relación. Así lo ha vislumbrado el jurista francés Ghestin, cuando ha puesto de manifiesto que la atención a la buena fe hace prevalecer el espíritu de la letra (la reciprocidad) y que no

25 Como consecuencia de crisis económica derivada de las difíciles condiciones impuestas a Alemania en el Tratado de Versalles, que puso fin a la Primera Guerra Mundial, la jurisprudencia desarrolló de forma significativa la cláusula general de la buena fe del $₫ 242$ BGB, como instrumento de justicia contractual (Miquel-González, 1997: 297 y ss.;Wieacker, 1982).

26 La buena fe limita la libertad de contratación en el art. 1:102 de los «Principles of European Contract Law» (PELC) y las partes tienen la obligación de actuar conforme a la misma, según su art. 1:201; por su parte el art. 1:202 regula el deber de colaborar. Éste se contempla en el «Draft Frame Common of Reference» (DFCR) (III. - 1:104) tras la buena fe y la honradez en los tratos (III. - 1: 103). Buena fe que aparece definida en el art. 2 (b) de la Propuesta de «Common European Sales Law» (CESL) como una norma de conducta caracterizada por la honradez, la franqueza y la consideración de los intereses de la otra parte de la transacción o de la relación en cuestión. A continuación su art. 3 incluye también el deber de cooperar. Junto a ellos se añade una larga lista de deberes de información.

27 Vid. Third Edition of UNIDROIT Principles of international Commercial Contracts, adoptados por el consejo de gobierno de unidroit en su 90 sesión, de 10 de mayo de 2011 en (http://www.unidroit. org/english/principles/contracts/main.htm). En su art. 5.1.2 se incluyen, entre las obligaciones implícitas en los contratos, la buena fe, la lealtad negocial e, incluso, el sentido común. Igualmente su art. 5.1.3 se refiere al deber de cooperación. 
basta la equivalencia entre las prestaciones, sino que se exige una igual posición de las propias partes (Ghestin, 2000, 90 y ss.). Se hace igualmente evidente el necesario desarrollo del ámbito de reciprocidad con ocasión del debate sobre la causa del contrato, puesto que también aquí se percibe un menor desarrollo del aspecto de los comportamientos por comparación con la función social atribuida a la causa (es decir, nuevamente en conexión con la socialidad). Con ocasión de los proyectos de reforma del Código Civil francés, Savaux ha propuesto una nueva denominación de la causa, de forma que describa correctamente el «elemento dinámico del contrato» (Savaux, 2011), que permitiría que tuviera lugar la dinámica bilateral entre institucionalidad y reciprocidad. Eso es precisamente la reciprocidad: la parte dinámica y viva de la relación jurídica. Permitir y reforzar el juego de adaptación mutua entre los dos componentes de la relación, considerada particularmente, y no sólo de forma social general, constituye ciertamente una exigencia de la justicia relacional.

Concluimos con la frase del civilista español J.A. Doral: «no toda convención es contrato sino la que origina una relación, establece las reglas y está provista de acción de cumplimiento contractual» (Doral-García, 2011: 112). Dicha frase, por un lado, recrea la doctrina de Ulpiano, contenida en su obra ad Edictum, ${ }^{28}$ pero, por otro, ahora resuena en el contexto de la justicia relacional, pudiéndose identificar en el ámbito de la socialidad la mención a la relación; en el ámbito de la institucionalidad la mención a las reglas; y en el ámbito de reciprocidad la mención a la acción, ya que contiene una referencia implícita a la causa. Efectivamente, Ulpiano, reclamando la protección mediante acción para las relaciones contractuales en las que la prestación se hubiera ya efectuado, afianzó la idea de una reciprocidad orientada a la institucionalidad, existiendo, en su estrecha conexión, una consideración de justicia. Sirva, pues, este retorno a Ulpiano, para insistir en el enfoque de justicia relacional del contrato, entendido como una relación jurídica, en un contexto de socialidad, cuyo criterio de justicia se vea reflejado continuamente en una vida nutrida por la reciprocidad de las partes y sostenida en el tiempo por la institucionalidad jurídica.

28 Concretamente, el libro IV, donde exponía la doctrina de los contratos bajo la rúbrica «de los pactos $y$ de las convenciones» (D. 2. 14. I. pr-3). 


\section{BIBLIOGRAFÍA}

Adams, J. y R. Brownsword (1987): «The Ideologies of Contract», Legal Studies.

- (2007): Understanding Contract Law, 5th. ed., London, Sweet \& Maxwell.

Atryah. P. S. (1990): Essays on Contract, Oxford, Oxford University Press.

Basozabal-Arrue, S. (2012): «Los deberes precontractuales de información después del DCFr. La Directiva 2011/83 y la Propuesta CESL» en CÁMARA, S. (dir.) (2012): La revisión de las normas europeas y nacionales de protección de los consumidores, Navarra,Thomson-Civitas.

Batiffol, H. (1968): «La crise du contrat», Archives de Philosophie du Droit, XIII, 1968, pp. 13-29.

Beale, H. y otros (2010): Cases, Materials and Text on Contract Law, Oxford, Hart Publising.

Bigwood, R. (2003): Explotative Contracts, Oxford, Oxford University Press.

Bosch-CAPDEVILA, E. (2012): «La anulación del contrato por explotación injusta en la Propuesta de Anteproyecto de ley de Modernización del Derecho de obligaciones y contratos» en BosCH-CAPDEVILA, E. (dir.) (2012): Nuevas perspectivas del Derecho contractual, Barcelona, Bosch.

Boudot, M. (2008): «La giustizia contrattuale in Francia», Europa e diritto privato, pp. 635-648.

Brownsword, R. (2006): Contract Law. Themes for the twenty-first century, Oxford, Oxford University Press.

BRUNI, L. (2012): Le nuove virtù del mercato nell'era dei beni comuni, Roma, Città Nuova.

- (2006): Reciprocità. Dinamiche di cooperazione, economia e societá civile, Torino, Bruno Mondadori.

CADIET, L. (2011): «Une justice contractuelle, l'autre» en GoubEaux, G. y otros (2011): Le contrat au début du XXI siècle. Études offertes à Jacques Ghestin, Paris, LGDJ.

Campbell, D. (2011): «Ian Macneil and the Relational Theory of Contract» en Campbell, D. (ed.) (2011): The Relational Theory of Contract: selected works of Ian Macneil, London, Sweet \& Maxwell-Thomson Reuters.

Campbell, D. y H. Collins (2003): «Discovering the Implicit Dimensions of Contracts» en CAMPBELl, D. y otros (eds.) (2003): Implicit Dimensions of Contracts. Discrete, Relational and Network Contracts, Oxford, Hart Publishing.

CAPPER, D. (1998): «Undue influence and unconscionability: a rationalization», Law Quarterly Review, 114, pp. 479-504. 
Carbonnier, J. (2001): Flexible droit, Paris, LGDJ.

Caro-GÁndara, R. (2013): «En la secular búsqueda europea de un paradigma de justicia contractual: el enfoque de justicia relacional» en Sánchez, S. (ed.) (2013): Derecho contractual comparado. Una perspectiva europea $y$ transnacional, Madrid, Civitas.

- (2011): «Good Corporate Governance and Relational Justice» en Economy, Finance and Management of Companies - Year 2011, Bratislava, Economy University of Bratislava.

СНАміе, J. F. (2012): «El principio general de reductio ad aequitatem por desequilibrio contractual», Revista de Derecho Privado (Colombia), 22, pp. 219-275.

Chazal, J. P. (2003): «Les nouveaux devoirs des contractants: est-on allé trop loin?» en JAMIN, C. y D. MAZEAUD (eds.): La nouvelle crise du contrat, Paris, Dalloz.

Chen-Wishart, M. (2010): Contract Law, Oxford, Oxford University Press.

Cossio, M. DE (1994): Frustraciones y desequilibrios contractuales, Granada, Comares.

Collins, H. (2003): «Discretionary Powers in Contracts» en CAMPBELl, D. y otros (eds.) (2003): Implicit Dimensions of Contracts. Discrete, Relational and Network Contracts, Oxford, Hart Publishing.

DONATI, P. (2006): Repensar la sociedad, Madrid, Eiunsa.

Doral-García, J. A. (2011): «La concepción del contrato en el Derecho europeo y su proyección en la propuesta de modernización» en AlbiEz-DoHrmanN, K. J. (dir.) (2011): Derecho privado europeo y modernización del Derecho contractual en España, Barcelona,Atelier.

EstebAN-DE LA Rosa, G. (2009): «El principio de cooperación en la contratación» en SÁnchez-Lorenzo, S. (ed.): Derecho contractual comparado. Una perspectiva europea y transnacional, Navarra, Thomson-Civitas.

Fabre-Magnan, M. (2001): «L'obligation de motivation en droit des contrats» en Goubeaux, G. y otros (2001): Le contrat au debut du XXI siècle. Études offertés à Jacques Ghestin, Paris, LGDJ.

Feinman, J. (2011): «The Reception of Ian Macneil's Work on Contract in the usa» en CAMpBell, D. (ed.) (2011): The Relational Theory of Contract: selected works of Ian Macneil, London, Sweet \& Maxwell-Thomson Reuters.

Flour, J. y otros (2012): Droit civil. Les obligations, t. 1, L'acte juridique, 15 éd., Paris, Dalloz-Sirey.

GÉraud-Llorca, E. (1994): «L'Introduction des bonnes moeurs dans le Code civil» en Les Bonnes Moeurs, Paris, PUF.

Gete-Alonso, M. C. (2008): Estudios sobre el contrato, Barcelona,Atelier. 
Ghestin, J. (2000): «Le contrat en tant qu'échange économique», Revue d'économie industrielle, 92, pp.81-100.

- (1990): «La notion de contrat», Recueil Dalloz, pp. 147-198.

- (1988): Traité de Droit Civil, Paris, LGDJ.

- (1981): «L'Utile et le juste dans le contrat»,Arch. Philo. Droit, 26, pp. 35-51.

Gilmore. G. (1995): The Death of Contract, Columbus, Ohio State University Press.

- (1974): The Death of Contract, Columbus, Ohio State University Press.

Gómez-Pomar, F. M. y M. Gili-Saldaña (2012): «El futuro instrumento opcional de Derecho contractual europeo: una breve introducción a las cuestiones de formación, interpretación, contenido y efectos», InDret 1/2012 [http:// www.indret.com/pdf/872_es.pdf].

Gordley, J. (2004): «Impossibility and Change of Unforeseen Circumstances», Amercian Journal of Comparative Law, 52, pp. 513-530.

Gordon, R. (1985): «Macaulay, Macneil and the Discovery of Solidarity and Power in Contract Law», Wisconsin Law Review, 1985, pp. 465-482.

Gounot, E. (1912): Le principe de l'autonomie de la volonté en droit civil. Contribution à l'étude critique de l'individualisme juridique, Thèse, Dijon.

Grossi, P. (2008): Europa y el Derecho, Barcelona, Crítica [traducción castellana de la obra original Grossi, P. (2007): L'Europa del Diritto, Roma-Bari, Laterza].

Grynbaum, L. y M. NiCOD (eds.) (2004): Le solidarisme contractuel, Paris, Economica.

HABERMAS, J. (1998a): Escritos sobre moralidad y eticidad, Barcelona, Paidós. - (1998b): Facticidad y Validez, Madrid, Trotta.

Habermas, J. y J. RaWls (1998): Debate sobre el liberalismo político, Barcelona, Paidós.

HartKamp, A.S. (2011): «Principles of European Contract Law» en HartKamp,A. S. y otros (2011): Towards a European Civil Code, The Hague, Kluwer.

Jauffret-Spinosi, C. (2006): «Théorie et pratique de la clause général en droit français et dans les autres systèmes juridiques romanistes» en Grundman, $\mathrm{S}$. y D. MAzEAud (eds.) (2006): General Clauses and Standars in European Contract Law, The Hague, Kluwer.

Josserand, J. (1934): "L'essor moderne du concept contractuel», Recueil d'études sur les sources du droit en l’honneur de François Gény, T. II, Vaduz-Paris, Topos-Duchemin.

Kartwrigt, J. (1991): Unequal Bargain. A Study of Vitiating Factors in the Formation of Contracts, Oxford, Claredom. 
KIMEL, D. (2007): «The Choice of Paradigm for Theory of Contract: Reflections on the Relational Model», Oxford Journal of Legal Studies, vol. 27, 2, pp. 233-255.

Lurger, B. (2011): «The Social Side of Contract Law and the New Principle of Regard and Fairness» en HARTKAMP, A.S. y otros (eds.) (2011): Towards a European Civil Code, La Haya, Kluwer.

- (2005): «The future of European Contract Law between freedom of Contract, Social Justice and Market Rationality», European Review of Contract Law, 1, pp. 442-510.

Macaulay, S. (2003): «The Real and the Paper Deal: Empirical Pictures of Relationships, Complexity and the Urge for Transparent Simple Rules» en CAMPBell, D. y otros (eds.) (2003): Implicit Dimensions of Contracts. Discrete, Relational and Network Contracts, Oxford, Hart Publishing.

- (1963a): «Non-contractual Relations in Business: A Preliminary Study», American Sociological Review, 28, pp. 55-69.

- (1963b): The Use and Non-use of Contracts in The Manufacturing Industry», The Practical Lawyer, 9, pp. 13-40.

MACNEIL, I. R. (2011): «The Relational Theory of Contract» en CAMPBELL, D. (ed.) (2011): The Relational Theory of Contract: selected works of Ian Macneil, London, Sweet \& Maxwell-Thomson Reuters.

- (2003): «Reflections on Relational Contract Theory after a Neo-classical Seminar» en CAMPBell, D. y otros (eds.) (2003): Implicit Dimensions of Contracts. Discrete, Relational and Network Contracts, Oxford, Hart Publishing.

- (1983): «Values in Contract: Internal and External», Nortbwestern University Law Review, 78, pp. 340-418.

- (1974): «The Many Futures of Contract», Soutbern California Law Review, 47, pp. 696-720.

- (1960): «Law in Society: An Introduction to Freedom of Contract», Cornell Law Quaterly, 47, pp. 177-192.

MACNEIL I. R. Y P. J. GUdEL (2001): Contracts. Exchange, Transactions and Relations. Cases and Materials, Nueva York, Foundation Press Edition (University Casebook Series).

Márquez-Prieto,A. (2012): «Justicia relacional y fraternidad» en Márquez-Prieto, A. (coord.) (2012): Fraternidad y justicia, Granada, Comares.

- (2010): Calidad ambiental de las relaciones laborales. Ensayo interdisciplinar, Granada, Comares.

- (2008): Repensar la justicia social, Pamplona,Thomson-Aranzadi.

Mazeaud, D. (1999): «Loyauté, solidarité, fraternité: la nouvelle devise contractuelle?» en Carbonnier, J. (1999): L'avenir du droit, Paris, Dalloz. 
- (1997): «Le régime de l'obligation de sécurité», Gazette du Palais, 2, pp. 1201-1212.

Miquel-GonZÁlez, J. M. (1997): «Cláusula general y desarrollo judicial del Derecho", Anuario de la Facultad de Derecho de la Universidad Autónoma de Madrid,pp. 297-326.

PICOD, Y. (2009): «Les projets français sur la réforme du droit des obligations», InDret, 4/2009 [http://www.raco.cat/index.php/InDret/article/ viewFile/141379/192890].

- (1993): Le juge et l'éxecution du contrat, Marsella, Aix-en-Provence, PU Aix-Marseille.

- (1989): Le devoir de loyauté dans l'éxecution du contrat, Paris, LGDJ.

- (1988): «L'obligation de cooperation dans l'exécution du contrat», JCP, pp. 3318-3332.

Posner, E. A. (1995): «Contract Law in the Welfare State: a Defense of the Unconscionability Doctrine, Usury Laws and Related Limitations of Freedom of Contracts»,Journal of Legal Studies, 24, pp. 283-319.

QuiÑones-Escámez, A. (2009): «Buena fe y lealtad contractual» en Sánchez, S. (ed.) (2009): Derecho contractual comparado. Una perspectiva europea $y$ transnacional, Navarra, Thomson-Civitas.

RÉMY, Ph. (1987): «Droit des contrats: questions, positions, propositions» en Le droit contemporain des contrats (Bilan et perspectives), Travaux et recherches de la Faculté de Sciences juridiques de Rennes, Economica, Paris.

RIPERT, G. (1925): La règle morale dans les obligations civiles, Paris, LGDJ.

SABorido-SÁncheZ, P. (2013): «La pervivencia de la relevancia jurídica de los propósitos o intereses de las partes en el contrato: la situación de la causa desde la perspectiva europea y desde los proyectos de reforma francés y español», InDret, 1/2013 [http://www.indret.com/pdf/953.pdf] .

- (2012): «Una visión crítica de la supresión de la causa en el nuevo derecho europeo de contratos. Su influencia en el ordenamiento jurídico español» en Bosch-Capdevila, E. (dir.) (2012): Nuevas perspectivas del Derecho contractual, Barcelona, Bosch.

SALVADOR-COderch, P. (2009): "Alteración de circunstancias en el art. 1213 de la Propuesta de Modernización de Código Civil en materia de obligaciones y contratos», InDret, 4/2009, pp. 1-60.

SavauX, E. (2011): «L'objet et la cause dans les projets francais de réforme du droit des contrats», documentación ofrecida a raíz de la conferencia impartida en el I Coloquio franco-español sobre el Derecho de Obligaciones, Santiago de Compostela, febrero 2011.

- (1997): La théorie génerale du contrat, mythe ou réalité?, Paris, LGDJ. 
Schiavone, A. (2005): Ius. l'Invenzione del diritto in Occidente, Torino, Giulio Einaudi.

Schulze, R. (2008): Common Frame of Reference and Existing EC Contract Law, München, Sellier.

STONe, R. (2002): The Modern Law of Contract, London, Cavendish Publishing Limited.

SuPIOT,A. (2005): Homo juridicus. Essai sur la fonction anthropologique du droit, Paris, Seuil.

Teubner, G. (2005): La cultura del Diritto nell'epoca della globalizzazione. L'emergere delle costituzioni civili [traduzzione e cura di Riccardo Prandini], Roma, Armando Editore.

- (2003): «Expertise as Social Institution: Internalising Third Parties into the Contract» en CAMPBELl, D. y otros (eds.) (2003): Implicit Dimensions of Contracts. Discrete, Relational and Network Contracts, Oxford, Hart Publishing.

Twigg-Flessner, C. (2008): «Precontractual duties-from the aquis to the Common Frame of Reference» en Schulze, R. (ed.) (2008): Common Frame of Reference and Existing EC Contract Law, Munich, Sellier.

Vincent-Jones, P. (2011): «The Reception of Ian Macneil's Work on Contract in the uK» en Campbell, D. (ed.) (2011): The Relational Theory of Contract: selected works of Ian Macneil, London, Sweet \& Maxwell-Thomson Reuters.

VolPe, F. (2004): La giustizia contrattuale tra autonomia e mercato, RomaNapoli, Edizioni Scientifiche Italiane.

WIEACKER, F. (1982): El principio general de la buena fe, Madrid, Civitas.

Wightman, J. (2003): «Beyond Custom: Contract, Contexts, and the Recognition of Implicit Understandings» en CAMPBELL, D. y otros (eds.) (2003): Implicit Dimensions of Contracts. Discrete, Relational and Network Contracts, Oxford, Hart Publishing. 